\title{
ETUDE DES DÉFORMATIONS DE LA HOULE AU VOISINAGE D'UNE JETÉE
}

par A. NIZERY

Ingénieur en Chef des Ponts et Chaussées

Chef du Service des Etudes et Recherches Hydrauliques

à l'Electricité de France

\section{COMMUNICATION PRESENTEE AU COMITE TECHNIQUE DE LA SOCIETE HYDROTECHNIQUE DE FRANCE DANS SA SEANCE DU 29 AVRIL 1947}

Dans une étude parue dans la «Revista de obras publicas » du $1^{\text {er }}$ janvier 1941, M. IRIBARREN, Ingénieur des Ponts et Chaussées d'Espagne, a donné une théorie sur les déformations de la houle au voisinage des côtes et des ouvrages des ports.

Cette théorie a pour but de donner aux ingénieurs des possibilités pratiques pour prévoir la forme en plan des crêtes de houle et l'amplitude de l'oscillation du niveau de la mer qui résulte en chaque point d'un plan d'eau de la proximité d'une plage ou d'ouvrages de protection. Elle est basée d'une part sur l'hypothèse de la houle trochoïdale, d'autre part sur des hypothèses approximatives concernant la propagation de l'énergie de la houle.

En ce qui concerne la forme des crêtes de houle en plan, elle ne soulève pas d'objection sérieuse. On peut cependant préférer la méthode exposée par M. GRIDEL, Ingénieur des Arts et Manufactures, dans les Annales des Ponts et Chaussées de Janvier-Février 1946, qui assimile la propagation de la houle à celle de la lumière et qui utilise pour le tracé des crêtes de houle la construction d'HUYGHENS.

L'étude de l'amplitude du mouvement vibratoire en chaque point du plan d'eau distingue deux natures d'expansion de la houle: I'expansion frontale et l'expansion latérale.

Nous n'insisterons pas sur l'expansion frontale. M. IRIBARREN admet que sur les plateformes côtières dont les pentes sont voisines de $1 / 100^{\circ}$ il s'effectue une espèce de compensation entre la réduction d'amplitude qui résulterait de la consommation d'énergie par frottement sur le fond et l'augmentation du creux qui serait provoquée par le raccourcissement de la longueur d'onde dû au relèvement progressif du fond. Cette hypothèse nécessiterait une vérification.

Quant à l'expansion transversale, elle se traduit, pour M. IRIBARREN, par l'allongement progressif des crêtes de houle provoqué par la divergence des normales à ces crêtes. Pour déterminer la variation de hauteur de la houle qui résulte de cette expansion, cet Ingénieur applique le principe de conservation de l'énergie aux longueurs des crêtes successives. Puis, passanit au cas particulier du pivotement de la houle autour d'un obstacle tel qu'une digue-abri, il admet qu'il se propage le long des crêtes déformées une onde positive de même longueur d'onde et de même célérité que la houle elle-

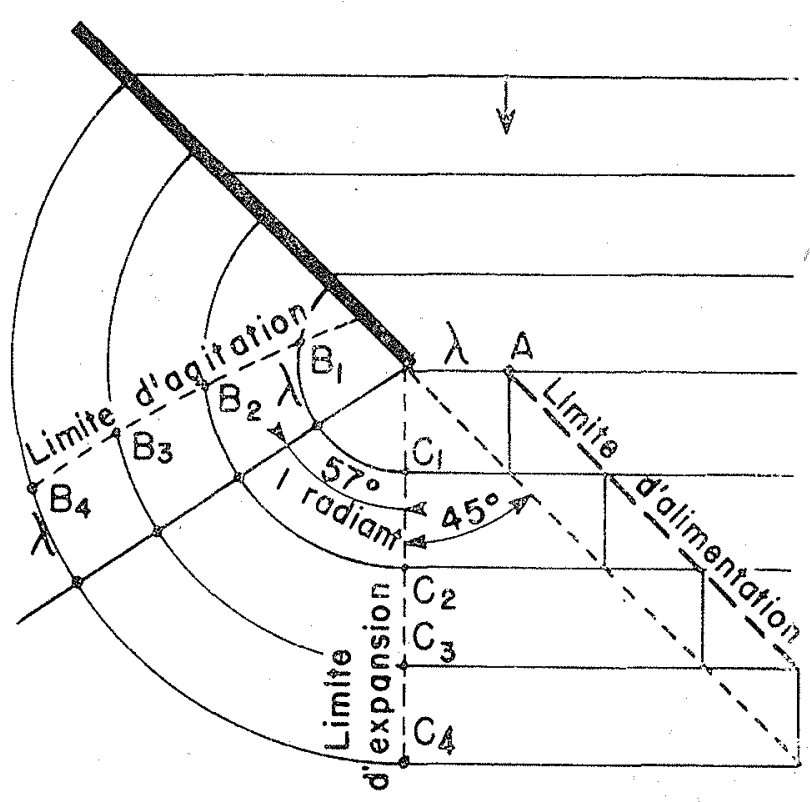

Fig. 1 
même. Cette onde positive part d'une droite passant par le musoir parallèle à la direction de propagation de la houle. L'énergie prélevée sur la houle par l'onde positive ci-dessus donne naissance à une onde négative qui se propage simultanément le long des crêtes non déformées.

M. IRIBARREN met ainsi en évidence une "limite d'alimentation » représentée par. une droite à $45^{\circ}$ sur la direction de propagation de la houle passant par le point A distant du musoir d'une longueur d'onde et une «limite dagitation passant par les points $B, B_{1}$ tels que les arcs $B_{1} C_{1}, B_{2} C_{2}$ sur les lignes de crêtes défor.. mées aient des longueurs égales à 2,3 fo:s la longueur d'onde.

L'hypothèse sur laquelle est basée la détermination de ces limites parait difficile à justifier. L'énergie qui provoque l'agitation à gauche de la ligne $C_{1} C_{2}$ dite ligne d'expansion ne peut. semble-t-il, venir que de la zone située à droite de cette ligne. Mais le mécanisme de transmission de cette énergie le long des crêtes déformées nous paraît bien difficile à accepter.

Nous avons cherché si, en utilisant l'analogie de la houle avec des phénomènes optiques suggérée par $M$. GRIDEL dans son mémoire précité, il était possible de résoudre d'une manière simple le problème limité au contournement par la houle d'un musoir en profondeurs constantes.

Le point de départ est évidemment l'hypothèse de HUYGENS-FRESNEL, d'après laquelle chaque point d'un front d'onde est un centre d'ébranlement à partir duquel l'ébranlement se propage dans toutes les directions en avant du front d'onde. Les physiciens ont longuement épilogué sur le fait que la vibration ne se transmet jamais en arrière du front d'onde et il a fallu attendre H. POINCARÉ, puis la théorie de KIRCHHOFF pour trancher le débat. En ce qui concerne la houle, la plupart des théories qui en ont été faites la réduisent à un phénomène à deux dimensions, mais il semble que rien ne s'oppose à ce que le principe d'HUYGHENS-FRESNEL lui soit intégralement appliqué.

Les oscillations de chaque point résulteraient de la composition en ce point de tous les ébranlements périodiques provenant d'un front d'onde situé en arrière et réciproquement les oscillations de ce point se transmettraient dans toutes les directions en avant du front d'onde au moment du passage de ce front au point considéré

Le problème du pivotement de la houle autour d'une jetée se réduirait alors tout simplement à un problème de diffraction au bord d'un grand écran.

Plaçons-nous dans le cas le plus simple où la jetée est placée parallélement à la direction générale des crêtes de houle au large. Nous n'exposerons pas ici la théorie de la diffraction; nous en indiquerons seulement les résultats généraux qui nous intéressent. Soit une source lumineuse $S$ et un écran J limité en $O$, pied de la perpendiculaire abaissée de $S$ sur J. Plaçons derrière le demi-écran J un écran $E$ parallèle à J (fig. 2).

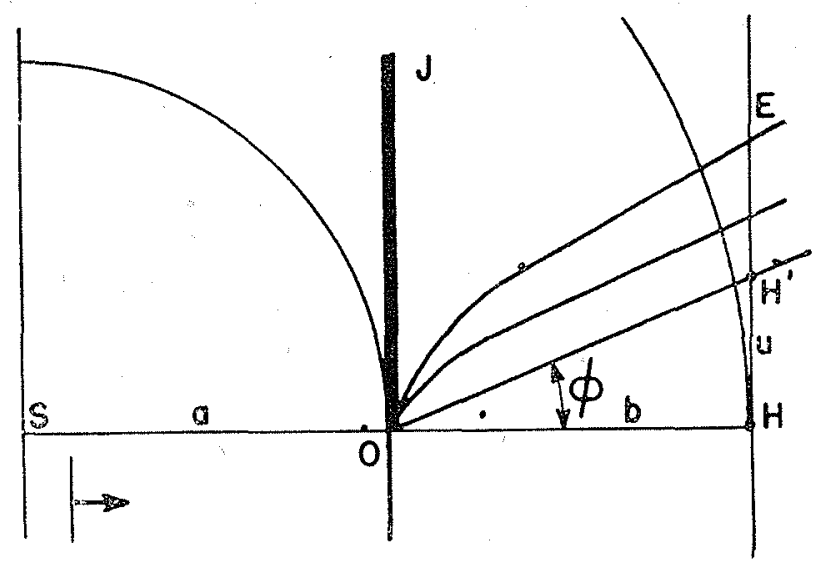

Fig. ?

La théorie et l'expérience montrent que l'on obtient :

$1^{\circ}$ à droite du point $\mathrm{H}$ pied de la perpendiculaire abaissée de $S$ sur $E$ une série de raies correspondant à des maxima et des minima d'intensité ;

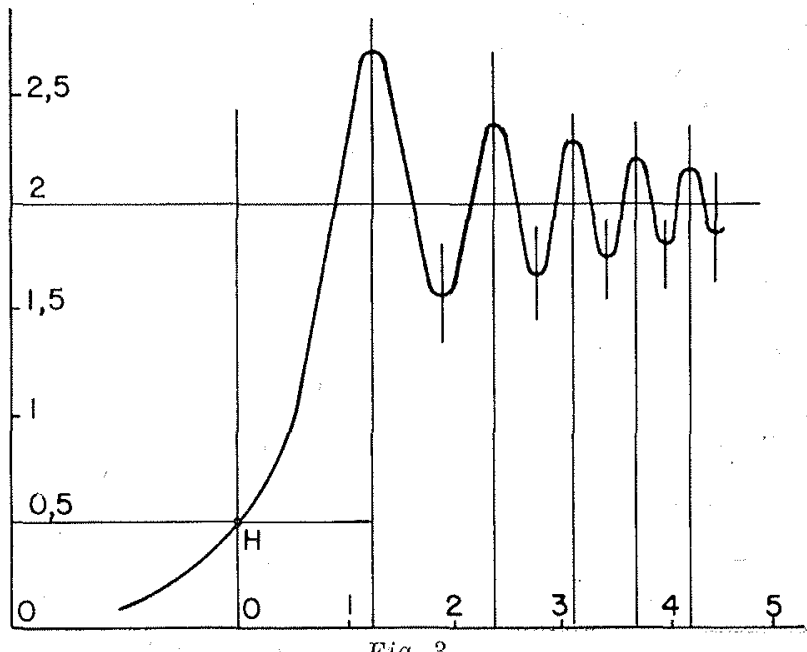


$2^{\circ}$ à gauche de $\mathrm{H}$ un éclairement décroissant rapidement de $\mathrm{H}$ vers la gauche.

Qualitativement, l'intensité se présente suivant la courbe de la fig. 3.

Nous laisserons de côté la partie à droite de $\mathrm{H}$. Ce qui nous intéresse c'est la pénétration de l'agitation dans la zone abritée, par conséquent la pénétration de la lumière à l'intérieur de l'ombre géométrique.

La loi de décroissance s'obtient facilement en utilisant les intégrales de FRESNEL.

Soit a la distance de la source $S$ au bord de l'écran $\mathrm{O}$.

Cherchons sur l'écran E placé à une distance b de l'écran J l'intensité lumineuse au point $\mathrm{H}^{\prime}$ situé à la distance u de $\mathrm{H}$.

$$
\begin{aligned}
& \text { Posons } V^{2}=\frac{2 a}{(a+b) b \text {; }} u^{2} \\
& \text { i.étant la longueur d'onde. }
\end{aligned}
$$

On sait que (1) l'intensité au point $\mathrm{H}^{\prime}$ est égale à $\mathrm{P}^{2}+\mathrm{Q}^{2}$

où

$$
\begin{gathered}
\mathrm{P}=\int_{-v}^{+\infty} \cos \frac{\pi \mathrm{V}^{2}}{2} \mathrm{dv}=\frac{1}{2}+ \\
\int_{0}^{v} \cos \frac{\pi \mathrm{V}^{2}}{2} d v=\frac{1}{2}+\mathrm{G}(\mathrm{v}) \\
\mathrm{Q}=\int_{-v}^{+\infty} \sin \frac{\pi \mathrm{V}^{2}}{2} \mathrm{dv}=\frac{1}{2}+ \\
\int_{0}^{v} \sin \frac{\pi \mathrm{V}^{2}}{2} d v=\frac{1}{2}+\mathrm{F}(\mathrm{v})
\end{gathered}
$$

$F$ et $G$ étant les intégrales de FRESNEL.

Si a tend vers l'infini ce qui correspond à des rayons parallèles ou à une houle au large à crêtes parallèles.

$$
V^{2} \text { tend vers } \frac{2 u^{2}}{b \lambda} \quad v=u \sqrt{\frac{2}{b \lambda}}
$$

On peut déterminer graphiquement les valeurs de I en utilisant la spirale de Cornu construite en portant en abscisses les valeurs de $G$ et en ordonnées les valeurs de $F$.

La racine carrée de l'intensité est donnée par le vecteur JP qui joint le point asymptotique

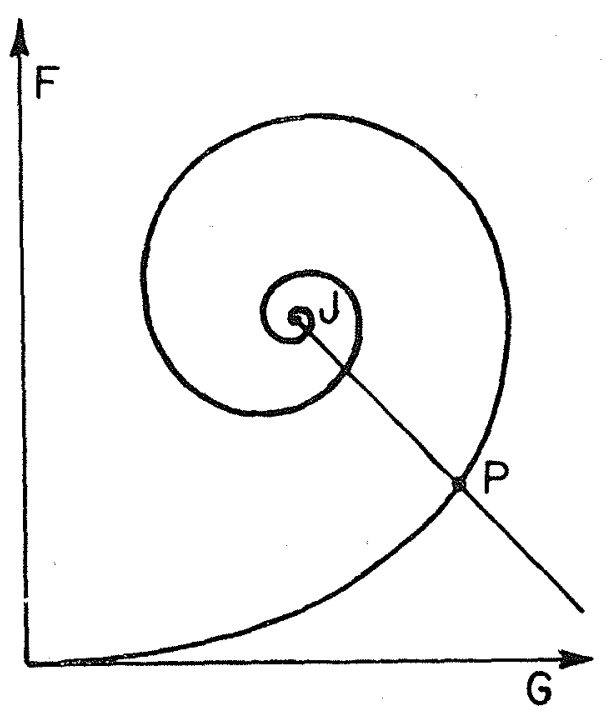

Fig. 4

correspondant à $V=+\infty$ au point de la courbe correspondant à

$$
v=u \sqrt{\frac{2}{b i}}
$$

$\operatorname{car} \overline{J P}^{2}=[G(\infty)-G(v)]+[F(\alpha)-F(v)]$

La fig. 5 représente la courbe donnant I en fonction de $V$.

Nous remarquons ensuite que l'intensité est la même pour tous les points où $V$ a la même valeur. Les courbes d'égale intensité sont donc les paraboles

$$
u^{2}=b \frac{V^{2}}{2}
$$

Ces paraboles ont leur sommet au bord de l'écran et sont tangentes à l'écran (fig. 2).

Leur axe est la droite qui passe par le bord de l'écran et qui est normale aux crêtes de houle.

Nous avons tracé dans cette famille de paraboles celles qui correspondent à une intensité de $1 / 4,1 / 10^{\circ}, 1 / 20^{\circ}, 1 / 50^{\circ}, 1 / 100^{\circ}$ de l'intensité initiale.

On lit d'abord sur la courbe I (v) la valeur de $v$ correspondant à la valeur de I sur la parabole d'égale intensité qu'on désire tracer, puis en posant $b=n \lambda$, on $a$ :

$$
u=v \lambda \sqrt{\frac{n}{2}}
$$




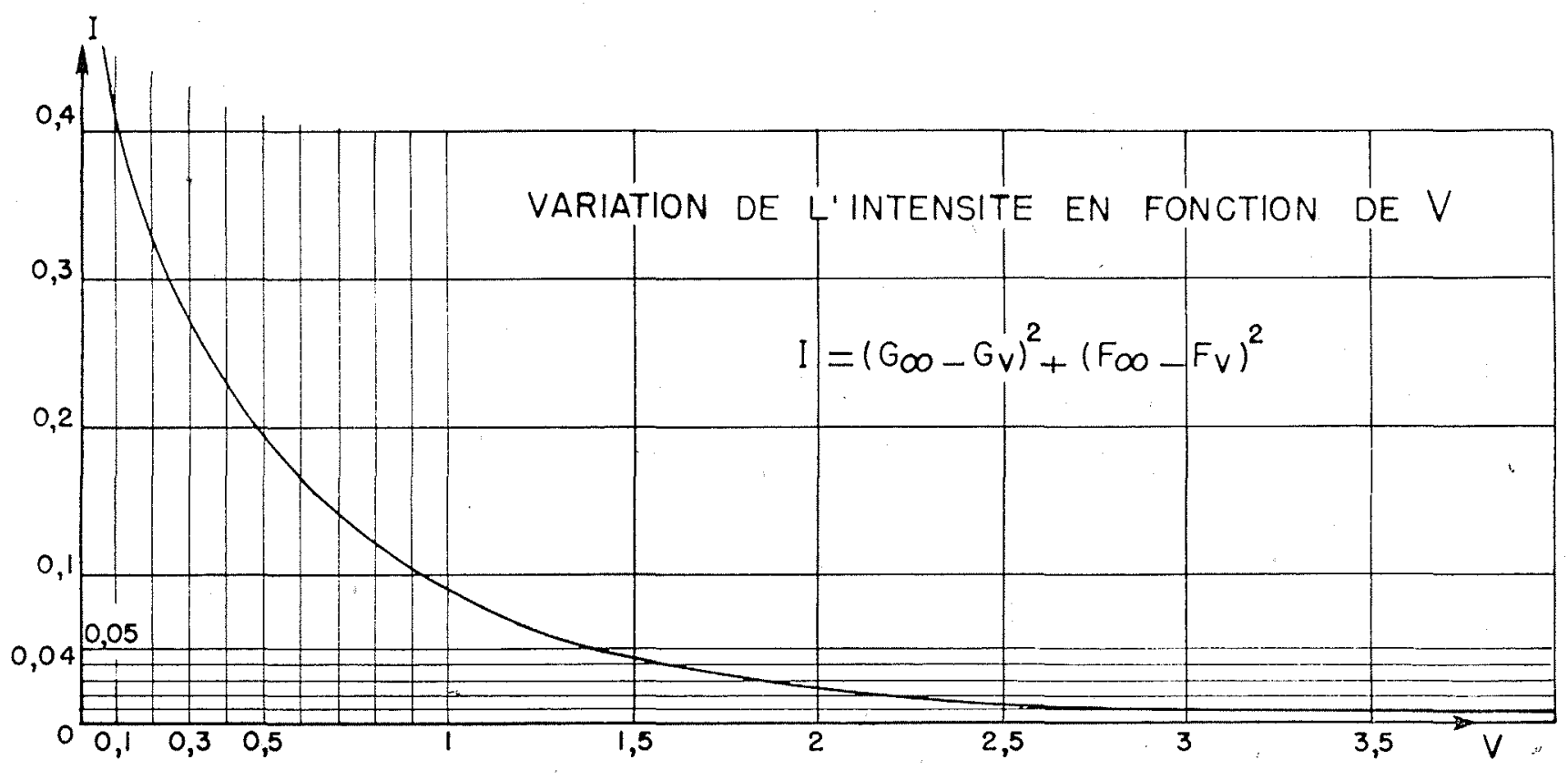

Fig. 5

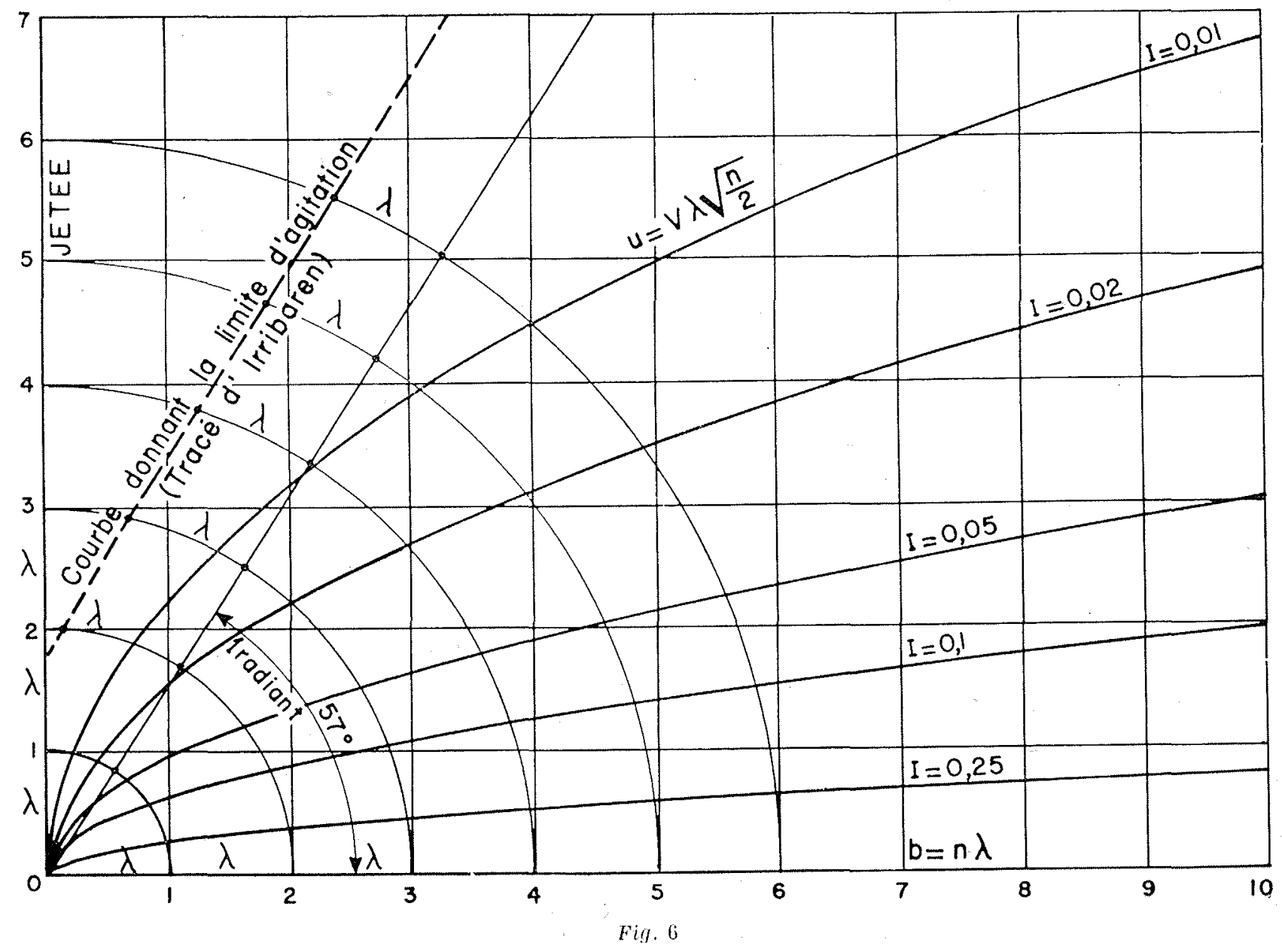


La fig. 6 donne le résultat obtenu. Nous y avons superposé le tracé de la limite d'agitation construite d'après la méthode de $M$. IRIBARREN.

On remarque que ce dernier tracé est nettement plus pessimiste que celui que donne la méthode optique.

Bien qu'aucune mesure systématique, $\mathrm{ni}$ aucune expérience de laboratoire ne permette encore de trancher le débat avec certitude, les constatations qualitatives que nous avons eu l'occasion de faire sur ce phénomène confirment l'allure générale qui ressort de l'application de la méthode optique. On constate en particulier que la houle se propage dans la totalité du bassin abrité par la jetée, contrairement à ce que l'on pourrait conclure de la théorie de M. IRIBARREN. Par ailleurs, le tracé de la limite pratique de l'agitation sensible rappelle nettement le résultat obtenu par la théorie optique.

Nous donnons à l'appui de ce qui précède la reproduction d'une photographie aérienne prise à la verticale d'une jetée d'un port colonial, avec toutes les imperfections d'un document qui n'était pas destiné à cet usage. On peut y ósserver en particulier une déformation des crêtes résultant de la variation de la profondeur. Néanmoins, nous avons tracé approximativement la direction générale des crêtes incidentes et la parabole théorique correspondant à $\frac{I}{I_{0}}=\frac{1}{10}$, celle-ci donne assez bien l'allure des courbes d'égale amplitude.

\section{Conclusion.}

Nous pensons qu'il serait du plus haut intérêt de vérifier dans la nature et au Laboratoire si la méthode simple que nous avons exposée ci-dessus donne des résultats quantitativement exacts. Elle fournirait, en effet, aux Ingénieurs, un instrument utile pour la conception de leurs projets.

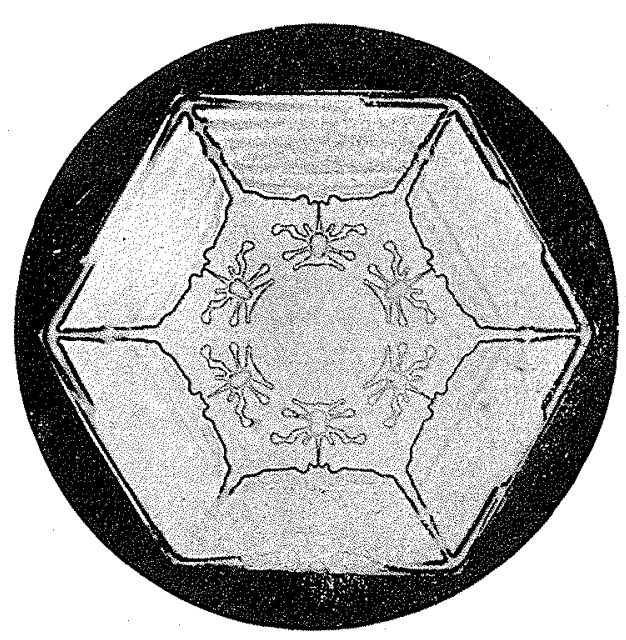




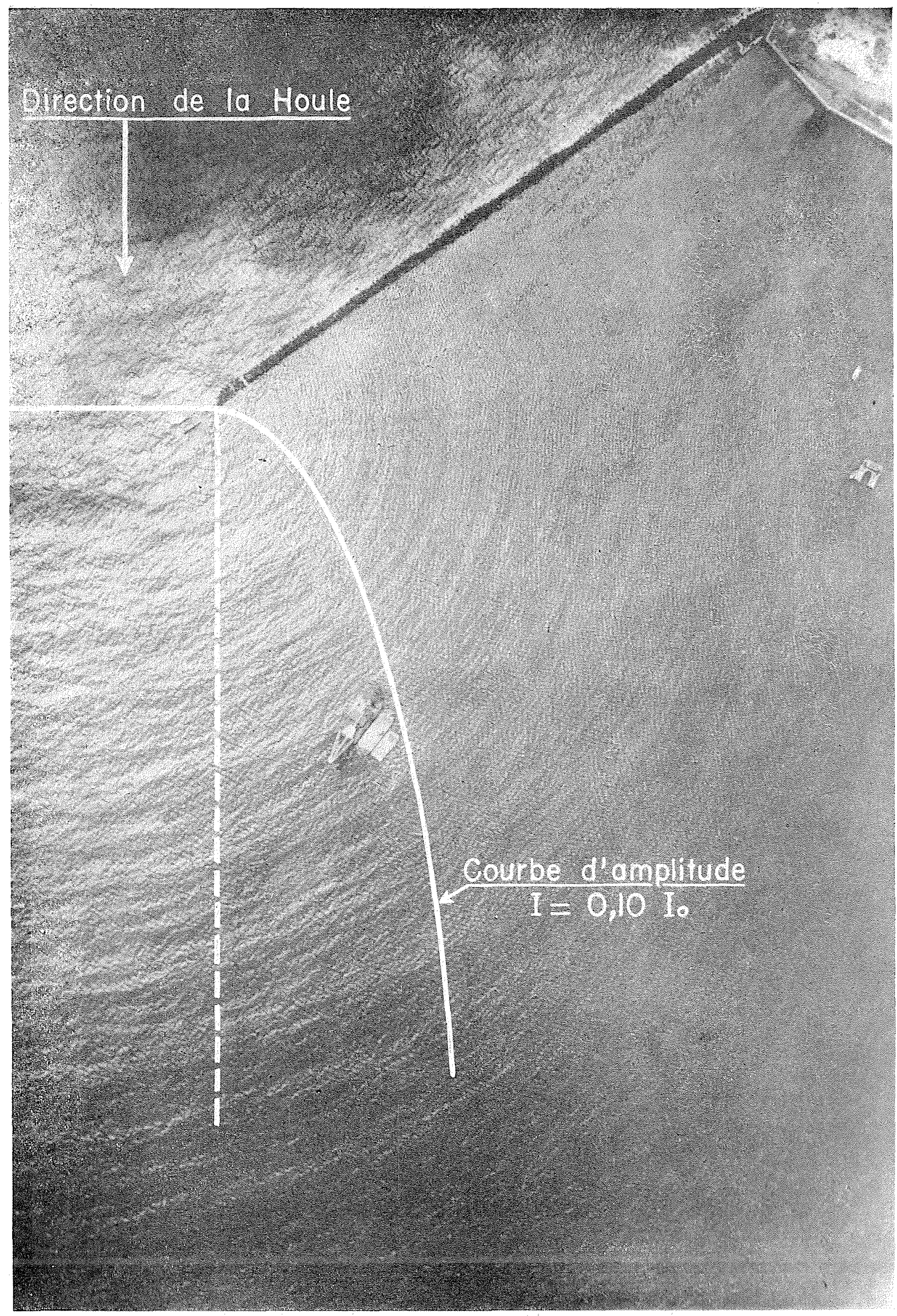

